\title{
Oral Recombinant Methioninase, Combined With Oral Caffeine and Injected Cisplatinum, Overcome Cisplatinum-Resistance and Regresses Patient- derived Orthotopic Xenograft Model of Osteosarcoma
}

\author{
TAKASHI HIGUCHI ${ }^{1,2,3}$, HIROMICHI OSHIRO ${ }^{1,2}$, KENTARO MIYAKE $^{1,2}$, \\ NORIHIKO SUGISAWA ${ }^{1,2}$, QINGHONG HAN ${ }^{1}$, YUYING TAN ${ }^{1}$, JUNHO PARK ${ }^{1,2}$, \\ ZHIYING ZHANG ${ }^{1,2}$, SAHAR RAZMJOOEI ${ }^{1}$, NORIO YAMAMOTO ${ }^{3}$, KATSUHIRO HAYASHI $^{3}$, \\ HIROAKI KIMURA ${ }^{3}$, SHINJI MIWA ${ }^{3}$, KENTARO IGARASHI $^{3}$, MICHAEL BOUVET $^{2}$, \\ SANT P. CHAWLA ${ }^{4}$, SHREE RAM SINGH ${ }^{5}$, HIROYUKI TSUCHIYA ${ }^{3}$ and ROBERT M. HOFFMAN ${ }^{1,2}$ \\ ${ }^{1}$ AntiCancer, Inc., San Diego, CA, U.S.A.; \\ ${ }^{2}$ Department of Surgery, University of California, San Diego, CA, U.S.A.; \\ ${ }^{3}$ Department of Orthopedic Surgery, Kanazawa University, Kanazawa, Japan; \\ ${ }^{4}$ Sarcoma Oncology Center, Santa Monica, CA, U.S.A.; \\ ${ }^{5}$ Basic Research Laboratory, National Cancer Institute, Frederick, MD, U.S.A.
}

\begin{abstract}
Background/Aim: Osteosarcoma is a recalcitrant neoplasm which occurs predominantly in adolescents and young adults. Recently, using a patient-derived orthotopic xenograft (PDOX) model of malignant soft-tissue sarcoma (STS), we showed that oral recombinant methioninase (orMETase), in combination with caffeine, was more efficacious than o-rMETase alone in inhibiting STS tumor growth. In the present report, we determined the efficacy of o-rMETase combined with oral caffeine on a cisplatinum (CDDP)resistant osteosarcoma PDOX model. Materials and Methods: Osteosarcoma PDOX models were randomly divided into seven treatment groups (6 mice in each group): untreated control; CDDP alone; o-rMETase alone; orMETase with caffeine; CDDP plus o-rMETase; CDDP plus caffeine; and CDDP plus o-rMETase with caffeine. Tumor size and body weight were measured throughout the treatment. Results: Tumors regressed after treatment with
\end{abstract}

Correspondence to: Robert M. Hoffman, Ph.D., AntiCancer, Inc., 7917 Ostrow Street, San Diego, CA 92111, U.S.A. Tel: +1 8586542555, Fax: +18582684175, e-mail: all@anticancer.com; Hiroyuki Tsuchiya, Department of Orthopaedic Surgery, Kanazawa University, 13-1, Takara-machi, Kanazawa 920-8641, Japan. Tel: +81 0762652374, email: tsuchi@med.kanazawa-u.ac.jp; Shree Ram Singh, Ph.D., Basic Research Laboratory, National Cancer Institute, Frederick, MD 21702, U.S.A. Tel: +1 3018467331, e-mail: singhshr@mail.nih.gov

Key Words: Osteosarcoma, PDOX, o-rMETase, caffeine, CDDP, regression.
CDDP plus o-rMETase with caffeine. Tumors treated with CDDP plus o-rMETase with caffeine also had the most necrosis. Conclusion: The combination of o-rMETase and caffeine together with first-line chemotherapy was efficacious for drug-resistant osteosarcoma and has clinical potential in the treatment of this highly-resistant neoplasm.

Osteosarcoma is the most frequent primary malignant bone tumor. Osteosarcoma is generally treated with a combination of various chemotherapy drugs and surgery (1). Cisplatinum (CDDP) has been the most effective therapeutic option for osteosarcoma, but outcome remains poor for most osteosarcoma patients when they develop chemoresistance (2).

Restriction of methionine (MET) selectively arrests cancer cells in $\mathrm{S} / \mathrm{G}_{2}$ with subsequent apoptosis (3). Cancer cells are highly dependent addicted on MET and their need of MET is restricted by a recombinant methioninase (rMETase). We have previously demonstrated that cancer cells arrested by MET restriction orally a are highly sensitive to caffeine because it induces mitotic catastrophe (4). We recently reported that oral rMETase (o-rMETase) inhibited tumor growth in a chemoresistant malignant soft-tissue sarcoma (STS) patient derived orthotopic xenograft (PDOX) model, and that combining o-rMETase with oral caffeine increased its efficacy (4). In addition, the tumor was regressed by adding this combination to first-line drug doxorubicin (DOX) (4). Thus, we consider that the administration of orMETase and caffeine together with chemotherapy is a promising strategy which could overcome chemoresistance. In this study, we analysed the efficacy of combining o- 
a

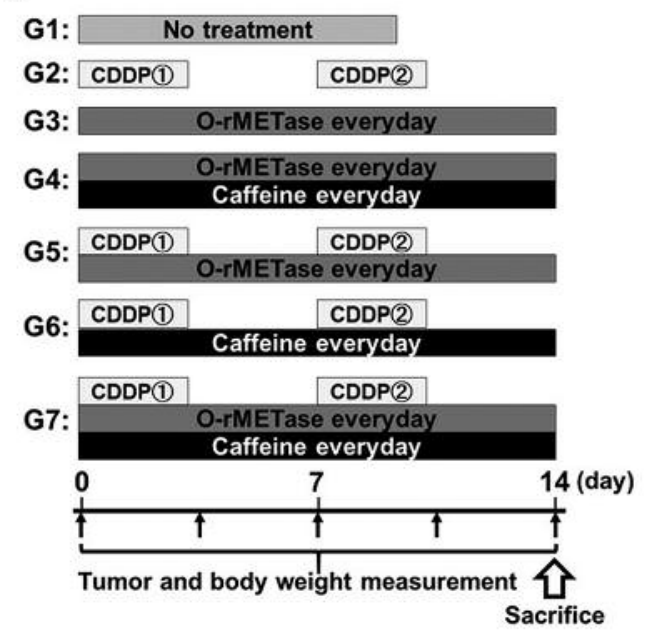

b

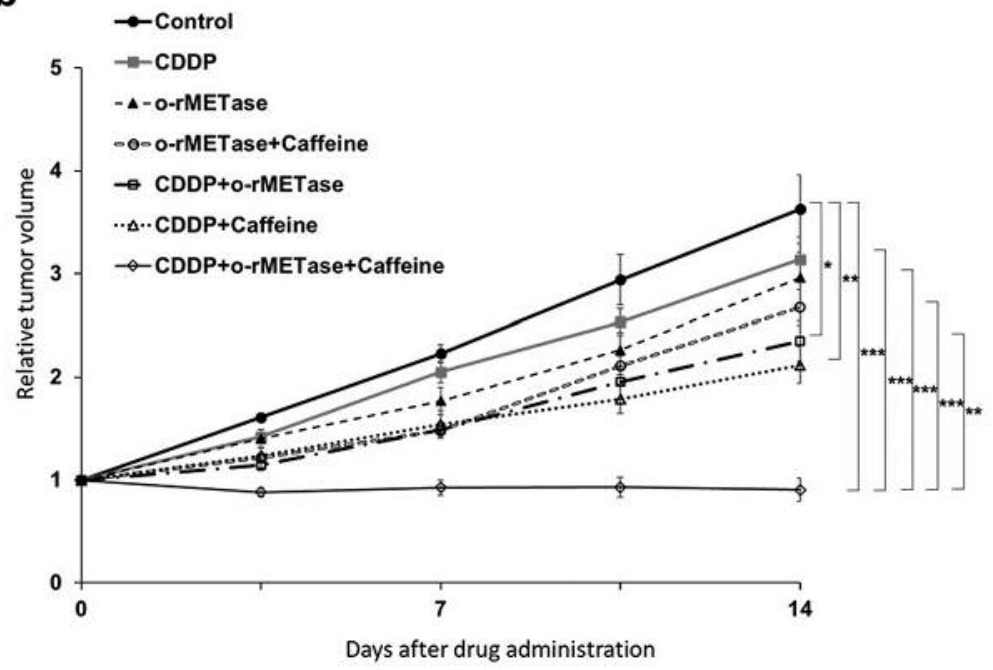

Figure 1. Efficacy of chemotherapy on the osteosarcoma-PDOX model. (a) Treatment regime. (b) Line graphs show tumor volume at each time point during treatment relative to the initial tumor volume for each treatment and control group. $N=6$ mice per treatment group. ${ }^{*} p<0.05 ; * * p<0.01$; $* * * p<0.001$. Error bars: \pm SEM.

rMETase and caffeine in overcoming CDDP-resistance in an osteosarcoma PDOX model.

\section{Materials and Methods}

Animals. Athymic nu/nu nude mice (AntiCancer, Inc., San Diego, CA, USA), 4-6 weeks old, were used (5). All experimental methods and data collection were carried based on our previous publications $(4,5)$. Anesthesia and analgesics were used to reduce any suffering of the animals as previously reported $(5,6)$. All mouse studies were performed at AntiCancer, Inc. An Institutional Animal Care and Use Committee (IACUC) protocol particularly approved for the present study were followed per the principles and procedures described in the National Institutes of Health (NIH) Guide for the Care and Use of Animals under Assurance Number A3873-1 (5, 6).

Patient-derived tumor. In this study, a fresh biopsy sample of a 14year-old boy with osteosarcoma in pelvis was used (6). The sample was processed at AntiCancer, Inc. The procedures of cutting small fragments and their subcutaneous implantation in nude mice have been previously described (5). No chemotherapy or radiotherapy was provided to the patient prior to biopsy (5). Written informed consent was obtained from the patient as part of the University of California, Los Angeles (UCLA) Institutional Review Board approved protocol (IRB\#10-001857) (6).

Establishment of an osteosarcoma PDOX model. The procedure for establishing osteosarcoma PDOX model including subcutaneouslyimplantation, harvesting of tumors, cutting them into small fragments, mice anesthesia and wound closure with nylon suture has been previously described (5-7).

Recombinant methionase (rMETase) production. rMETase production has been described previously (8).

Treatment regimen for the osteosarcoma PDOX model. The osteosarcoma PDOX mouse models were randomly divided into 7 groups (6 mice each) (Figure 1a). Mice were treated for 2 weeks using the following drugs: G1, untreated control; G2, CDDP (6 $\mathrm{mg} / \mathrm{kg}$, intraperitoneal (i.p.) injection, once a weekl) alone (6); G3, o-rMETase (100 units/day for 14 consecutive days, oral administration) alone; G4, o-rMETase + caffeine $(100 \mathrm{mg} / \mathrm{kg} /$ day for 14 consecutive days, oral administration); G5, CDDP + o-rMETase; G6, CDDP + caffeine; G7, CDDP + o-rMETase + caffeine. Treatment started once tumors were reached $100 \mathrm{~mm}^{3}(6)$. Tumor volume and body weight measurements were performed twice a week (6-8). The formula to calculate tumor volume has been previously described (68). Data are presented as mean \pm standard error of the mean (SEM).

Histological analysis. Samples were fixed, sectioned and stained as previously reported (6-8).

Statistical analysis. All statistical analyses were performed as previously reported (6-8). All $p$-values were two-sided and $p$-values of less than 0.05 were regarded as statistically significant.

\section{Results}

Efficacy of the tested drugs on the osteosarcoma-PDOX. No significant inhibition of osteosarcoma PDOX was obtained when tumors were treated with CDDP alone, o-rMETase alone, or with o-rMETase + caffeine compared with control: Control vs. CDDP: $p=0.91$; vs. o-rMETase: $p=0.48$; vs. orMETase + caffeine; $p=0.18$. In contrast, mice treated with $\mathrm{CDDP}+\mathrm{o}$-rMETase, CDDP + caffeine, and CDDP + orMETase + caffeine showed significant inhibition of tumor growth compared to control: Control vs. CDDP $+\mathrm{o}-$ rMETase: $p=0.02 ; v s$. CDDP + caffeine: $p=0.003$; $s$. CDDP + o-rMETase + caffeine: $p<0.001$. Tumor regression was 

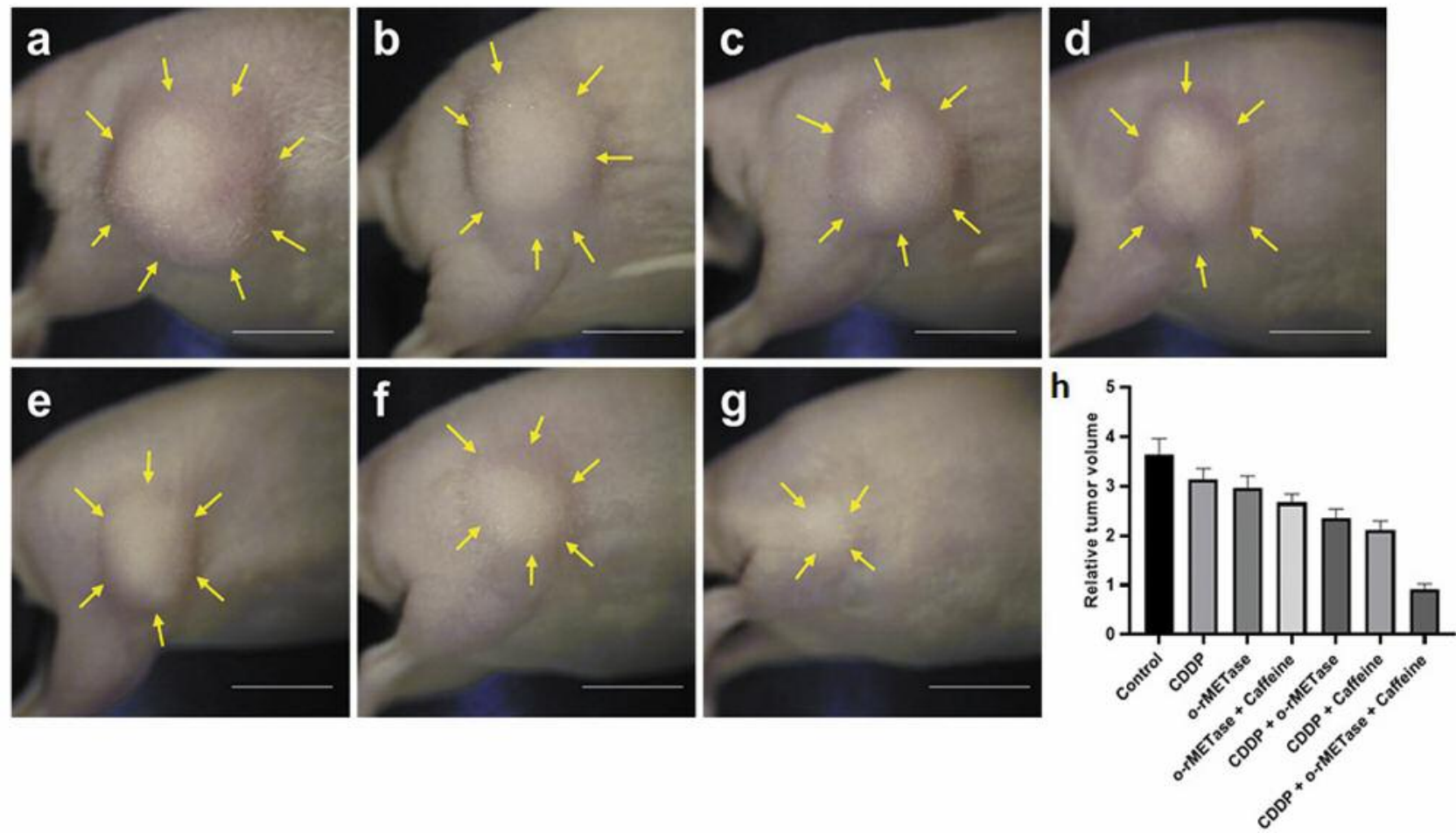

Figure 2. Images of representative osteosarcoma-PDOX model from each treatment group at the end of treatment. (a) Untreated control. (b) CDDP alone. (c) o-rMETase alone. (d) o-rMETase + caffeine. (e) CDDP + o-rMETase. (f) CDDP + caffeine. (g) CDDP + o-rMETase + caffeine. Arrows show the margins of the tumors. Scale bars: $10 \mathrm{~mm}$. (h) relative tumor volume for each drug treatment on day 14.

observed only in the mice treated with CDDP + o-rMETase + caffeine. Significant differences were found in tumor growth between the CDDP + o-rMETase + caffeine treated group, and all the other treatment groups except for the CDDP + caffeine treated group: CDDP + o-rMETase + caffeine vs. CDDP: $p<0.001$; vs. o-rMETase: $p<0001$; $v s$. orMETase + caffeine: $p<0.001 ; v s$. CDDP + rMETase: $p=0.008$ ) (Figures $1 \mathrm{~b}$ and 2).

Histology of the osteosarcoma-PDOX. The untreated tumor (control) was specifically composed of viable highly-dense tumor cells which had spindle-shaped cells with extensive pleomorphism, many mitotic figures, and bizarre appearing cells with hyperchromatic nuclei (6). In addition, osteoid laid down in a lace-like pattern in between the tumor cells was also seen (6). Tumors treated with CDDP, o-rMETase alone, o-rMETase and caffeine, CDDP plus o-rMETase, CDDP plus caffeine comprised viable tumor cells, although the tumor cell density was lower compared to the control group, and degenerative scar change in the stroma cells was also observed. Extensive tumor necrosis with increased inflammatory cell infiltration was seen in tumors treated with CDDP plus o-rMETase and caffeine. The strong anti-tumor efficacy of CDDP plus o-rMETase and caffeine treatment on
osteosarcoma-PDOX tumor was demonstrated (Figure 3a-g).

Effect of treatment on body weight. Mean body weight of the mice significantly increased in control $(p<0.001)$ and orMETase treated group $(p<0.001)$. However, no significant difference was found in body weight between pre- and posttreatment in any other treatment group (Figure 3h). No other side effects or animal deaths were observed in any treatment group.

\section{Discussion}

Osteosarcoma is a frequently occurring malignant bone tumor in young people. Osteosarcoma patients have a very poor 5-year survival rate, because osteosarcoma is a highly aggressive neoplasm that shows poor response to chemotherapy drugs (9). It is often treated with a combination of chemotherapy drugs, and surgery (1). CDDP has been used as an effective drug for osteosarcoma, however, outcome remains poor for most osteosarcoma patients once they develop chemoresistance $(2,10,11)$.

The present results suggest that the osteosarcoma-PDOX is methionine-dependent since it responds to o-rMETase. Combining caffeine with o-rMETase can increase its efficacy 

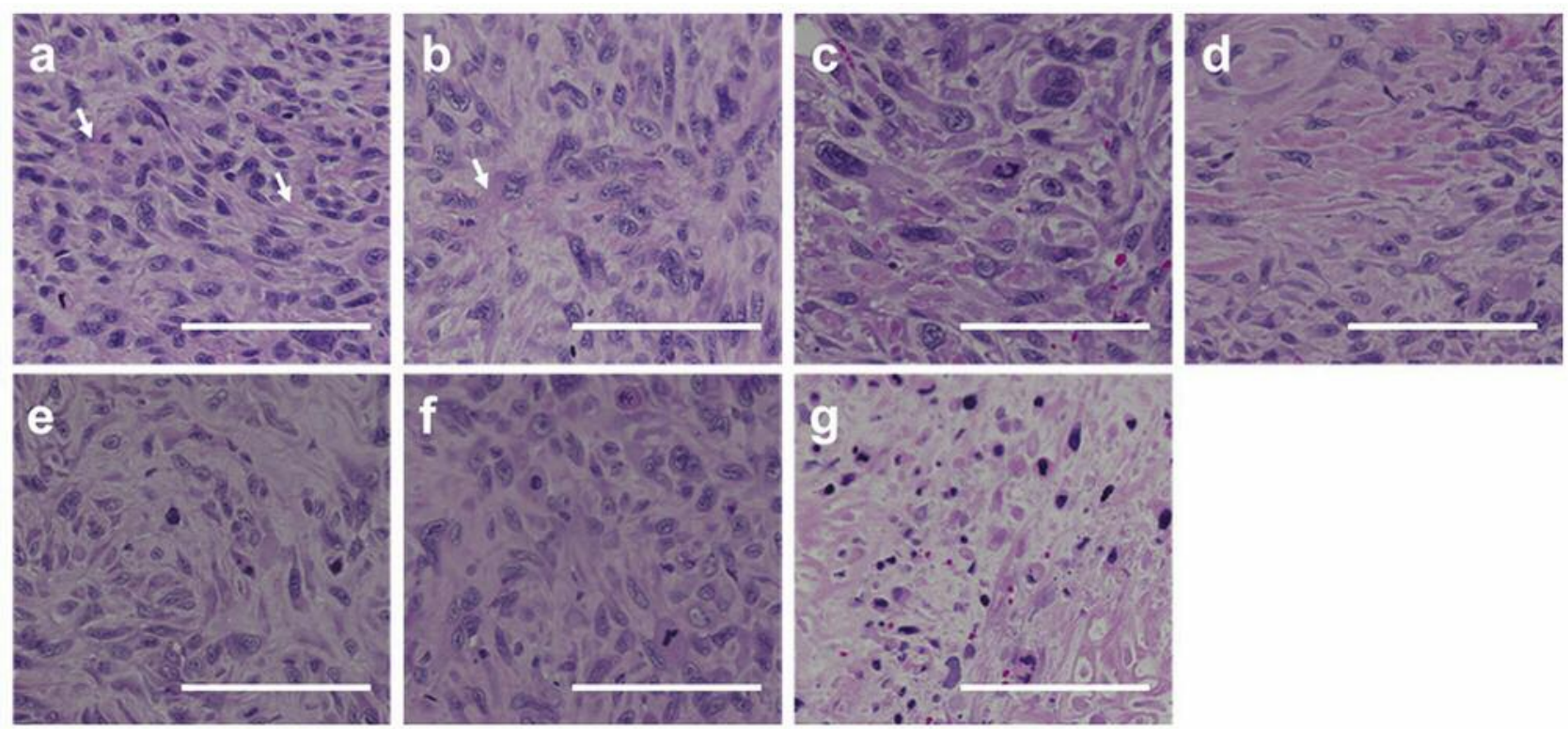

h

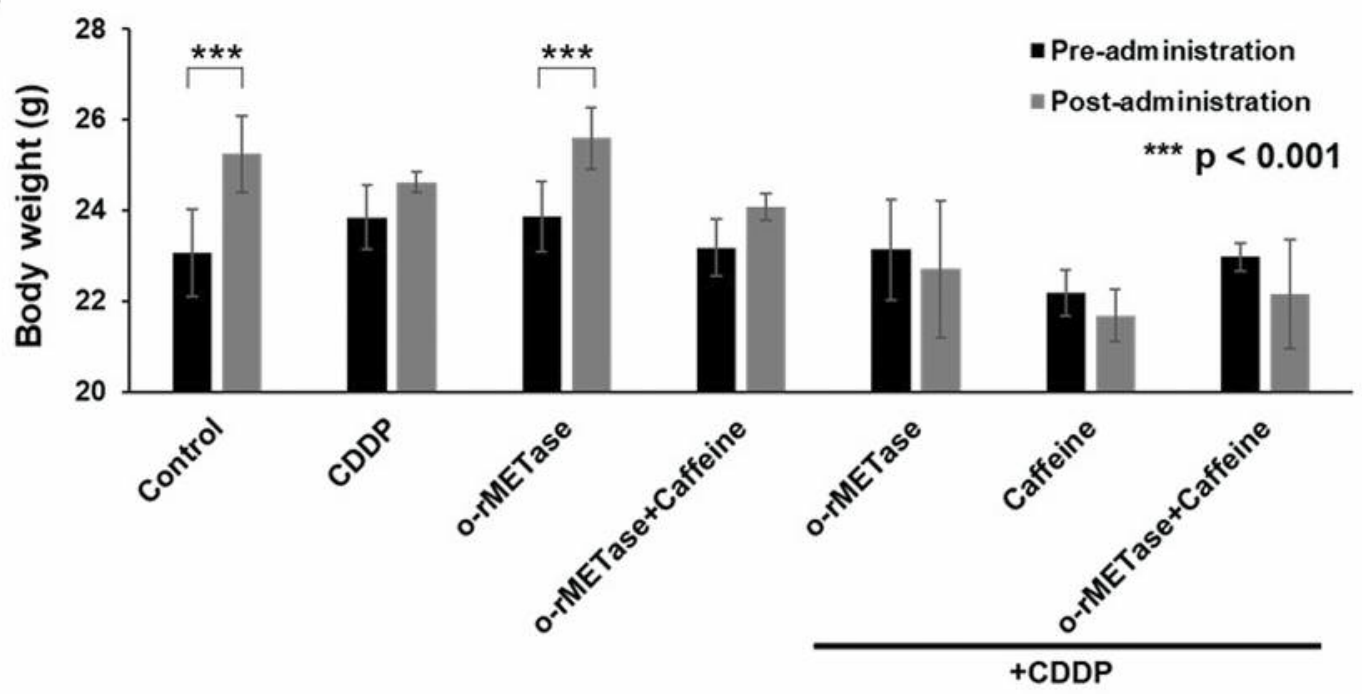

Figure 3. Tumor histology and mouse body weight. (a) untreated control. (b) CDDP. (c) o-rMETase. (d) o-rMETase + caffeine. (e) CDDP + orMETase. (f) CDDP + caffeine. (g) CDDP + o-rMETase + caffeine. Arrows indicate tumor osteoid. Scale bars: $100 \mu \mathrm{m}$. (h) Mouse body weight $(\mathrm{g})$ is depicted in bar graphs for each treatment group or control group at pre- and post-treatment times. ${ }^{*} *$ * $<<0.001 ;$ Error bars: \pm SEM.

against the osteosarcoma-PDOX tumor, which was further strongly enhanced by adding CDDP.

CDDP, DOX, and high-dose methotrexate are generally given to osteosarcoma patients either alone or in combination, as a first-line treatment $(1,6)$. Survival of osteosarcoma patients has improved greatly in the past few decades due to these chemotherapy drugs. However, the survival rate decreases to $30-40 \%$ if the tumor is CDDP resistance $(2,12)$.

Cancer cells are MET-dependent (Hoffman effect) because they excessively utilize MET for aberrant transmethylation reactions (12), which is similar to a situation when cancer cells utilize excess amount of glucose (Warburg effect) (13, 14). PET imaging of cancer with [11C]MET provides a robust signal compared to fluorodeoxyglucose [18F]FDG suggesting that the Hoffman effect is considerably prominent compared to the Warburg effect (15).

We recently reported that combining caffeine to orMETase increased the efficacy of rMETase by lowering plasma L-MET levels compared to o-rMETase alone (4), and 
addition of o-rMETase and caffeine in combination with DOX resulted in regression of a DOX-resistant synovial sarcoma (SS) PDOX (4). In the present study, o-rMETase and caffeine combination with CDDP was effective and induced regression of the osteosarcoma-PDOX tumor, perhaps due to induction of mitotic catastrophe by caffeine on rMETase S/G2-arrested cancer cells (4). Combining orMETase and caffeine with CDDP could overcome CDDPresistance in osteosarcoma without observable toxicity.

In conclusion, the present study determined that o-rMETase could inhibit tumor growth of osteosarcoma-PDOX and combining o-rMETase with caffeine could increase its efficacy. Adding CDDP to this combination regressed the osteosarcomaPDOX tumor and overcame CDDP-resistance in osteosarcoma, suggesting an important clinical potential of this strategy.

\section{Conflicts of Interest}

AntiCancer Inc. uses PDOX models for contract research. TH, HO, $\mathrm{KM}, \mathrm{NS}, \mathrm{QH}, \mathrm{YN}, \mathrm{JP}, \mathrm{ZZ}, \mathrm{SR}$, and RMH are or were unsalaried associates of AntiCancer Inc. The authors have no other competing financial interests.

\section{Authors' Contributions}

Conception and design: TH and RMH. Acquisition of data: TH, HO, $\mathrm{KM}, \mathrm{NS}, \mathrm{QH}, \mathrm{YN}, \mathrm{JP}, \mathrm{ZZ}, \mathrm{SR}$. Analysis and interpretation of data: TH, HO, KM, NS, QH, YN, JP, ZZ, SR, NY, KH, HK, SM, KI, MB, SPC, SRS, HT, and RMH. Study supervision: RMH. Manuscript writing/revision of the manuscript: TH, RMH and SRS. All Authors reviewed the manuscript.

\section{References}

1 Misaghi A, Goldin A, Awad M and Kulidjian AA: Osteosarcoma: a comprehensive review. SICOT J 4: 12, 2018. PMID: 29629690. DOI: $10.1051 / \operatorname{sicotj} / 2017028$

2 Pasello M, Michelacci F, Scionti I, Hattinger CM, Zuntini M, Caccuri AM, Scotlandi K, Picci P and Serra M: Overcoming glutathione S-transferase $\mathrm{P} 1$-related cisplatin resistance in osteosarcoma. Cancer Res 68(16): 6661-6668, 2008. PMID: 18701490. DOI: 10.1158/0008-5472.CAN-07-5840

3 Hoffman RM: Development of recombinant methioninase to target the general cancer-specific metabolic defect of methionine dependence: a 40-year odyssey. Expert Opin Biol Ther 15(1): 2131, 2015. PMID: 25439528. DOI: 10.1517/14712598.2015. 963050

4 Higuchi T, Kawaguchi K, Miyake K, Han Q, Tan Y, Oshiro H, Sugisawa N, Zhang Z, Razmjooei S, Yamamoto N, Hayashi K, Kimura H, Miwa S, Igarashi K, Chawla SP, Singh AS, Eilber FC, Singh SR, Tsuchiya H and Hoffman RM: Oral recombinant methioninase combined with caffeine and doxorubicin induced regression of a doxorubicin-resistant synovial sarcoma in a PDOX mouse model. Anticancer Res 38(10): 5639-5644, 2018. PMID: 30275182. DOI: 10.21873/anticanres.12899

5 Igarashi K, Kawaguchi K, Li S, Han Q, Tan Y, Murakami T, Kiyuna T, Miyake K, Miyake M, Singh AS, Eckardt MA, Nelson SD, Russell TA, Dry SM, Li Y, Yamamoto N, Hayashi K, Kimura H, Miwa S, Tsuchiya H, Singh SR, Eilber FC and
Hoffman RM: Recombinant methioninase in combination with doxorubicin (DOX) overcomes first-line DOX resistance in a patient-derived orthotopic xenograft nude-mouse model of undifferentiated spindle-cell sarcoma. Cancer Lett 417: 168-173, 2018. PMID: 29306021. DOI: 10.1016/j.canlet.2017.12.028

6 Higuchi T, Miyake K, Oshiro H, Sugisawa N, Yamamoto N, Hayashi K, Kimura H, Miwa S, Igarashi K, Chawla SP, Bouvet M, Singh SR, Tsuchiya H and Hoffman RM: Trabectedin and irinotecan combination regresses a cisplatinum-resistant osteosarcoma in a patient-derived orthotopic xenograft nudemouse model. Biochem Biophys Res Commun 513(2): 326-331, 2019. PMID: 30955860. DOI: 10.1016/j.bbrc.2019.03.191

7 Igarashi K, Kawaguchi K, Kiyuna T, Miyake K, Miyake M, Li Y, Nelson SD, Dry SM, Singh AS, Elliott IA, Russell TA, Eckardt MA, Yamamoto N, Hayashi K, Kimura H, Miwa S, Tsuchiya H, Eilber FC and Hoffman RM: Temozolomide combined with irinotecan regresses a cisplatinum-resistant relapsed osteosarcoma in a patient-derived orthotopic xenograft (PDOX) precisiononcology mouse model. Oncotarget 9(8): 7774-7781, 2018. PMID: 29487690. DOI: 10.18632/oncotarget. 22892

8 Tan Y, Xu M, Tan X, Tan X, Wang X, Saikawa Y, Nagahama T, Sun X, Lenz M and Hoffman RM: Overexpression and largescale production of recombinant L-methionine-alpha-deaminogamma-mercaptomethane-lyase for novel anticancer therapy. Protein Exp Purif 9(2): 233-245, 1997. PMID: 9056489. DOI: 10.1006/prep.1996.0700

9 Meyers PA: Muramyl tripeptide (mifamurtide) for the treatment of osteosarcoma. Expert Rev Anticancer Ther 9: 1035-1049, 2009. PMID: 19671023. DOI: 10.1586/era.09.69

10 Siddik ZH: Cisplatin: mode of cytotoxic action and molecular basis of resistance. Oncogene 22(47): 7265-7279, 2003. PMID: 14576837. DOI: $10.1038 /$ sj.onc. 1206933

11 Keremu A, Aini A, Maimaitirexiati Y, Liang Z, Aila P, Xierela $\mathrm{P}$, Tusun A, Moming H and Yusufu A: Overcoming cisplatin resistance in osteosarcoma through the miR-199a-modulated inhibition of HIF-1 $\alpha$. Biosci Rep pii: BSR20170080, 2017. PMID: 28442599. DOI: 10.1042/BSR20170080

12 Miwa S, Takeuchi A, Ikeda H, Shirai T, Yamamoto N, Nishida H, Hayashi K, Tanzawa Y, Kimura H, Igarashi K and Tsuchiya $\mathrm{H}$ : Prognostic value of histological response to chemotherapy in osteosarcoma patients receiving tumor-bearing frozen autograft. PLoS One 8(8): e71362, 2013. PMID: 23977028. DOI: 10.1371/ journal.pone.0071362

13 Stern PH and Hoffman RM: Elevated overall rates of transmethylation in cell lines from diverse human tumors. In Vitro 20(8): 663-670, 1984. PMID: 6500606.

14 Hoffman RM: The wayward methyl group and the cascade to cancer. Cell Cycle 16(9): 825-829, 2017. PMID: 28318368. DOI: $10.1080 / 15384101.2017 .1304330$

15 Warburg O: On the origin of cancer cells. Science 123(3191): 309314, 1956. PMID: 13298683. DOI: 10.1126/science. 123.3191 .309

16 Mitamura K, Yamamoto Y, Norikane T, Hatakeyama T, Okada M and Nishiyama Y: Correlation of (18)F-FDG and (11)C-methionine uptake on PET/CT with Ki-67 immunohisto-chemistry in newly diagnosed intracranial meningiomas. Ann Nucl Med 32(9): 627633, 2018. PMID: 30032455. DOI: 10.1007/s12149-018-1284-6

Received July 1, 2019

Revised July 9, 2019

Accepted July 10, 2019 